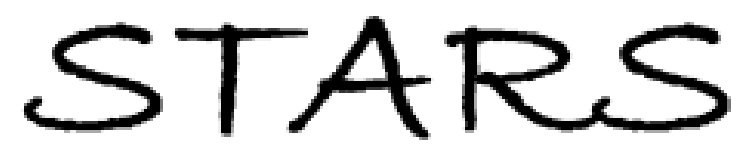

University of Central Florida

STARS

$1-1-2005$

\title{
Role of ions in pool boiling heat transfer of pure and silica nanofluids
}

Denitsa Milanova

University of Central Florida

Ranganathan Kumar

University of Central Florida

Find similar works at: https://stars.library.ucf.edu/facultybib2000 University of Central Florida Libraries http://library.ucf.edu

This Article is brought to you for free and open access by the Faculty Bibliography at STARS. It has been accepted for inclusion in Faculty Bibliography 2000 s by an authorized administrator of STARS. For more information, please contact STARS@ucf.edu.

\section{Recommended Citation}

Milanova, Denitsa and Kumar, Ranganathan, "Role of ions in pool boiling heat transfer of pure and silica nanofluids" (2005). Faculty Bibliography 2000s. 5486.

https://stars.library.ucf.edu/facultybib2000/5486

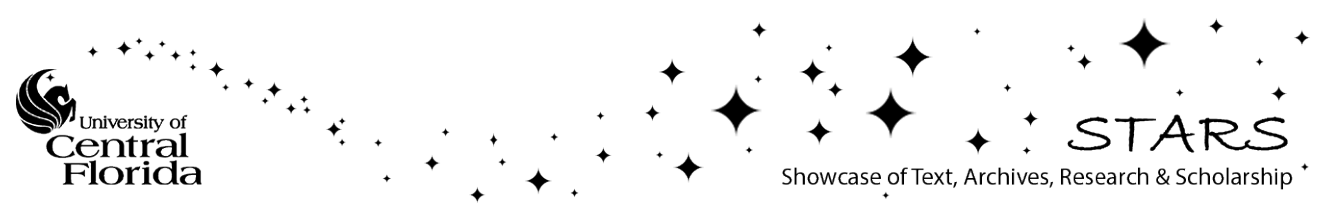




\title{
Role of ions in pool boiling heat transfer of pure and silica nanofluids
}

\author{
Denitsa Milanova and Ranganathan Kumar ${ }^{a}$ \\ Department of Mechanical, Materials and Aerospace Engineering, University of Central Florida, \\ Orlando, Florida 32816
}

(Received 29 June 2005; accepted 6 October 2005; published online 29 November 2005)

\begin{abstract}
Heat transfer in silica nanofluids at different acidity and base is measured for various ionic concentrations in a pool boiling experiment. Nanosilica suspension increases the critical heat flux 3 times compared to conventional fluids. The 10-nm particles possess a thicker double diffuse layer compared to $20-\mathrm{nm}$ particles. The catalytic properties of nanofluids decrease in the presence of salts, allowing the particles to cluster and minimize the potential increase in heat transfer. Nanofluids in a strong electrolyte, i.e., in high ionic concentration, allow a higher critical heat flux than in buffer solutions because of the difference in surface area. The formation and surface structure of the deposition affect the thermal properties of the liquid. (C) 2005 American Institute of Physics.
\end{abstract}

[DOI: $10.1063 / 1.2138805$ ]

Several investigators ${ }^{1-3}$ have reported a significant enhancement in critical heat flux when nanoparticles of sizes less than $50 \mathrm{~nm}$ are dispersed in pure water in a pool boiling environment. Such fluids with nanoparticle suspensions are called nanofluids. The anomalous thermal properties and the suspension characteristics make nanofluids promising for nanotechnology based heat transfer applications such as electronic cooling and heat exchangers.

Even at very low concentrations, these nanofluids possess high thermal conductivity. In the presence of nanoparticles, particle-particle and particle-molecule interactions play a major role in increasing the thermal conductivity of the nanofluids which enhances the heat transfer. A few possible factors to enhance the thermal conductivity are discussed in published literature, including the Brownian motion of nanoparticles, ordered arrangement of liquid molecules in the vicinity of nanoparticles, ballistic heat transfer mode in nanoparticles, and clustering of nanoparticles. ${ }^{4-6}$

In this letter, we show that the heat transfer characteristics are even more pronounced when the pool boiling experiment is performed in a strong electrolyte. The objective of this work is to systematically study the effect of the negatively charged silica nanoparticle suspension in boiling conditions in water for different particle size, particle concentration, and under various $p \mathrm{H}$ conditions and ionic concentration.

The experiments were done using a suspended nichrome $(\mathrm{NiCr})$ wire immersed in water with varying $p \mathrm{H}$. The wire of length $46 \mathrm{~mm}$ and $0.32 \mathrm{~mm}$ in diameter was horizontally suspended in a pool of water in a glass beaker and tightly attached to a copper lead wire, and then soldered. The data for the boiling curve was obtained by slowly increasing the current and recording the voltage. Depending on the chemical composition of the liquid, the wire could suddenly break or glow due to a jump in voltage. The boiling curve was generated by using a calibration curve from a known temperature-resistance relationship for $\mathrm{NiCr}$ (Ref. 7) and obtaining the heat flux from

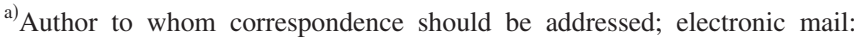
rnkumar@mail.ucf.edu
}

$$
q=I^{2} R_{w} / \pi D L
$$

where $q$ is the heat flux, $R_{w}$ is the wire resistance, $D$ is the wire diameter, and $L$ is the wire length. The experiments were conducted with $250 \mathrm{ml}$ of water completely immersing the wire. The boiling process evaporated about $5 \mathrm{ml}$ of water during the experiment, i.e., $<2 \%$, which had no significant effect on the temperature or heat flux measurements. Silicon (IV) oxide is a commercial product of Alfa Aesar, and was acquired in a colloidal suspension. The weight percentage of 10 and $20 \mathrm{~nm}$ is $15 \%$ and $40 \%$, respectively. Nanofluids used in the pool boiling experiment were prepared by stirring nanosilica in different acidity and salt content base liquids for about $30 \mathrm{~min}$. Experiments were run after a good dispersion of nanoparticles was achieved.

The uncertainly in the resistance measurements from the multimeter is $0.459 \%$. The soldering of the wires at both ends adds uncertainty to the length of the whole wire and attributes to the largest uncertainty in the heat flux, which is $5.8 \%$. The uncertainty in the $T-R$ curve fit for nichrome is estimated to be $30^{\circ} \mathrm{C}$.

Since particle suspensions are stable at different $p \mathrm{H}$ solutions depending on the particle characteristics, our initial investigation focused on the effect of $p \mathrm{H}$ in pure water. Pool boiling is a mode of heat transfer that occurs on a surface submerged in a pool of initially quiescent fluid with a change in phase from liquid to vapor. Referring to the boiling curve of water at different $p \mathrm{H}$, photographs of $p \mathrm{H} 7$ are provided in Fig. 1 to illustrate the physical mechanisms taking place at various heat flux. The different $p \mathrm{H}$ solutions were prepared using either $\mathrm{HCl}$ or $\mathrm{NaOH}$ so that the initial ionic concentration of the $\mathrm{H}+$ or the $\mathrm{OH}-$ ions can be calculated. Initially, as the current is passed through the wire, the heat transfer mode changes from natural convection to the saturated boiling regime where bubbles begin to coalesce. As the heat flux is further raised beyond what is known as the critical heat flux (CHF), a sudden large increase in temperature occurs. With the wire being blanketed by mostly vapor, there is again a linear rise of wire temperature with an increase of heat flux, until a point is reached when the wire breaks. Near CHF, the heating surface is covered by rising vapor columns in the form of jets with countercurrent liquid jets flowing downward as shown in Fig. 1. Based on the fact that CHF is limited by the removal rate of bubbles which carry away the 


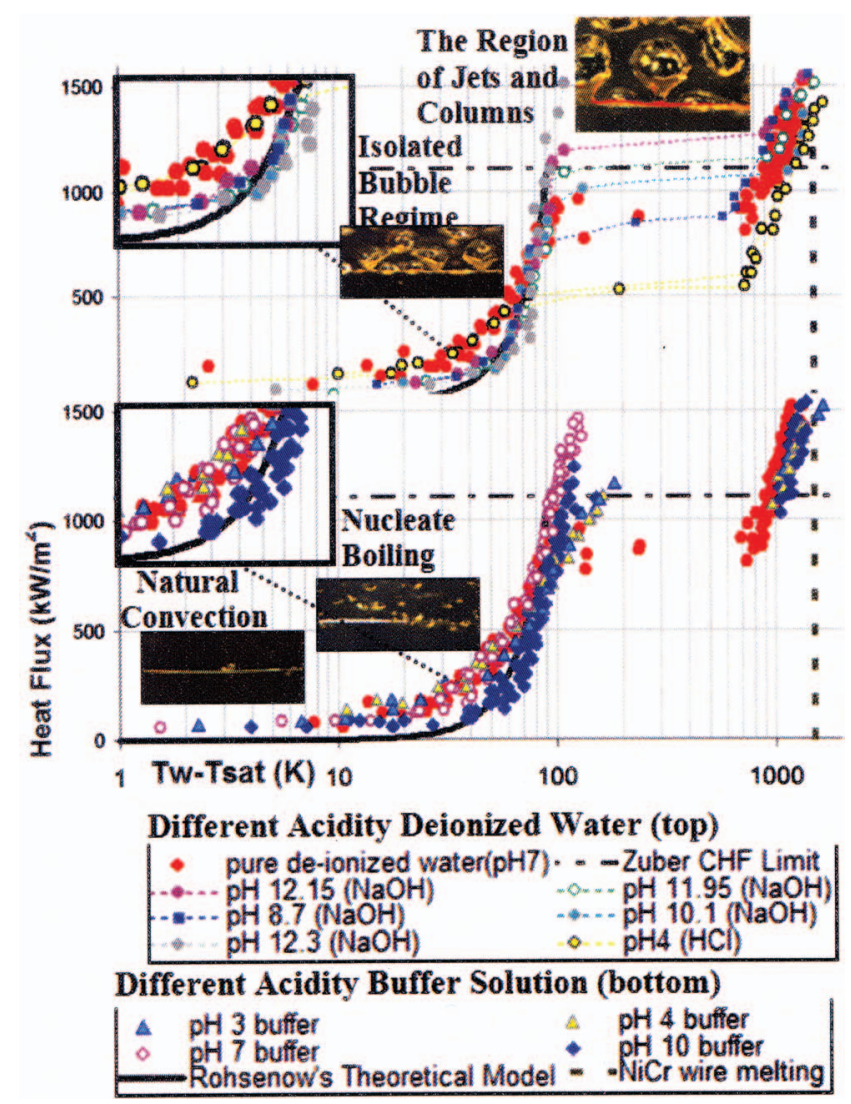

FIG. 1. (Color) Heat flux vs wire superheat at different $\mathrm{pH}$. The photographs at different heat flux show different boiling regimes.

heat in the form of heat of vaporization, Zuber ${ }^{8}$ obtained CHF for pure water as

$$
q_{\max }^{\prime \prime}=0.131 h_{f g} \rho_{\nu}^{1 / 2}(g \sigma \Delta \rho)^{1 / 4},
$$

where $h_{f g}, \sigma$, and $\Delta \rho$ are the heat of vaporization, surface tension, and the density difference between the phases, respectively. The data obtained in this paper compare well with the CHF given in Eq. (2) as well as with the saturated boiling regime preceding CHF as given by Rohsenow's ${ }^{9}$ wellestablished correlation

$$
\begin{aligned}
q_{\text {flux Ros }}(\Delta T):= & \mu_{I} h_{f g}\left[\frac{\sigma}{g_{1}(\Delta \rho)}\right]^{-0.5}\left(\frac{1}{C_{\text {sf }}}\right)^{1 / 0.33} \\
& \times \operatorname{Pr}^{-1.7 / 0.33}\left[\frac{c_{p l}(\Delta T)}{h_{f g}}\right]^{1 / 0.33},
\end{aligned}
$$

where $\mu_{f}$ is the fluid viscosity, $\Delta T$ is the temperature superheat, $\mathrm{Pr}$ is the Prandtl number, and $C_{\mathrm{sf}}$ is taken to be 0.045 .

As seen in Fig. 1, for different $p \mathrm{H}$, the heat flux where the horizontal curve appears (this happens due to a sudden jump in the wire resistance) decreases with increasing acidity. This is the point where the wire begins to glow. The nichrome wire dipped in an acidic solution begins to melt at a much earlier stage compared to a basic solution. Metal glowing usually occurs at high temperatures (above $800{ }^{\circ} \mathrm{C}$ ). The flow of electrons from the positive electrode (anode) in the system to the negative one (cathode) seems to be vital in determining the vapor phase behavior during the process of electrolysis. The positively charged hydrogen ion goes to the anode which has a negatively charged surface, takes an electron (oxidation), and becomes a neutrally charged hydrogen atom $(\mathrm{H})$. Hydrogen atoms combine to form a hydrogen gas

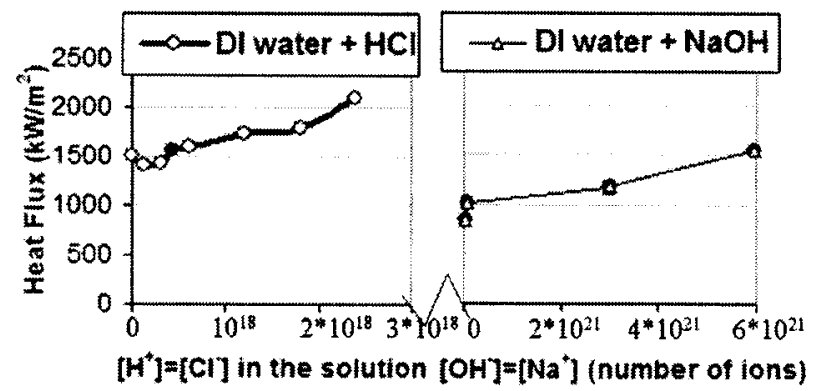

FIG. 2. $\mathrm{CHF}$ as a function of the number of dissociated ions (a) $\mathrm{H}^{+}$and $\mathrm{Cl}^{-}$ and (b) $\mathrm{Na}+$ and $\mathrm{OH}-$ in deionized water.

$\left(\mathrm{H}_{2}\right)$ which bubbles up from the anode and the wire. A similar phenomenon was seen as in Ref. 10. When the amount of $\mathrm{HCl}$ is increased, no jump in temperature is noticed, and the CHF value starts to increase; however, the wire breaks before glowing.

The number of ions was calculated by multiplying the number of moles (mass/molarity) by Avogadro number. Since the anode is the positive lead wire and its surface is negatively charged by electrons, there is a point at which oxidation and formation of hydrogen gas is no longer possible due to a high concentration of positive ions, which leads to a depletion of $e-$. In Fig. 2, this transition appears when the number of ions exceeds $5 \times 10^{17}$. Beyond this point, the $\mathrm{H}+$ ions remain free and carry part of the applied current, bypassing the wire and giving it "longer life," hence, the wire is able to carry more current, increasing CHF.

In the case of increasing $\mathrm{NaOH}$ base [Fig. 2(b)], cations give off their excess electrons (the process of reduction), and when they become neutral, oxygen gas $\mathrm{O}_{2}$ is formed. Thus, the hydrogen and oxygen gases determine the different stages of the boiling curve and the extent of the glowing or the absence of it. Additional insight can be obtained by conducting the heat transfer experiments using buffer solutions. In the case of a weak electrolyte, pure buffer solutions at different $p \mathrm{H}$ exhibit a jump in resistance and subsequent glowing at all $p \mathrm{H}$ except 7.0 (Fig. 1) Buffers are weak electrolytes and give off free ions at a very controlled rate, therefore, it is reasonable to expect wire melting when a temperature of $\sim 800{ }^{\circ} \mathrm{C}$ is reached. That means the sudden jump in temperature always occurs at about $1100 \mathrm{~kW} / \mathrm{m}^{2}$, which is the Zuber CHF limit for pure solutions. In the case of the $p \mathrm{H} 7$ buffer solution, there is a balance of dissociated positive and negative ions and the wire does not glow. The boiling characteristics are different for $p \mathrm{H} 7$ deionized (DI) water in that the dissociated oxygen atoms allow the wire to glow.

Silica nanofluid is basically colloidal silica, i.e., fine particles dispersed in water, a state between bulk and molecular dispersed systems. The surface of silicon dioxide is negatively charged by the hydroxyl groups which occupy the space between the oxygen atoms during its manufacturing to achieve mutual repulsion and stability.

It has been already established by Vassallo et al. ${ }^{3}$ that in silica nanosuspensions, the critical heat flux can be increased by over $100 \%$. In Figs. 3 and 4, it is shown that it can be improved to $300 \%$ depending on the particle size and the $p \mathrm{H}$ level.

It is important to investigate the process of polymerization (Fig. 3) and dispersion (Fig. 4) for the heat transfer analysis because polymerization (as in the buffer solution in Fig. 3) leads to lower surface area to volume ratio and, con- 


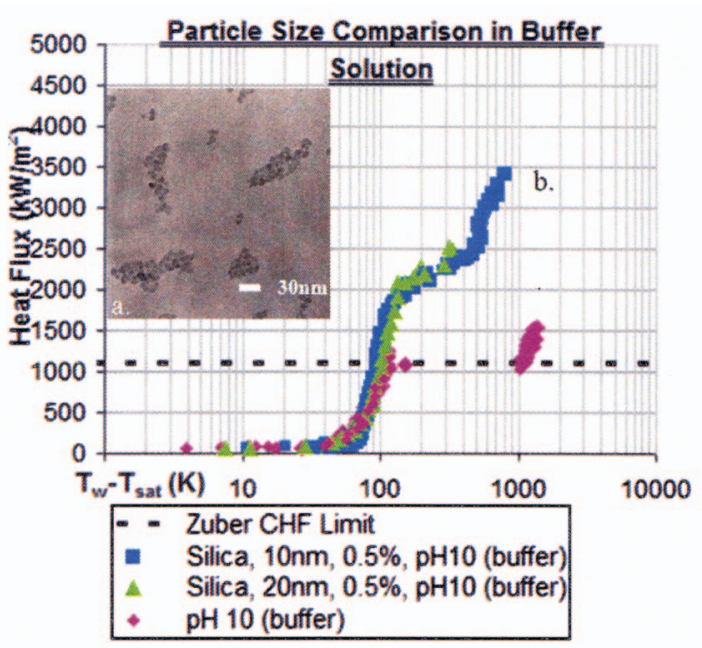

FIG. 3. (Color) (a) Agglomeration characteristics as seen in TEM of silica in buffer $p \mathrm{H}$ 10. (b) Pool boiling CHF for 10 and $20 \mathrm{~nm}$ particles in $p \mathrm{H} 10$ solution.

sequently, to lower heat flux. According to the general theory of polymerization, ${ }^{11}$ in the presence of salts $(p \mathrm{H} 7-10)$ aggregation and gelling occur due to a decrease in mutual repulsion. This effect is noticed in the TEM image in Fig. 3. In the absence of salts for $p \mathrm{H} 7-10$, as shown in the TEM image in Fig. 4, the repulsive barriers are strong enough to guarantee particle growth without aggregation.

The double diffuse layer surrounding the particle is calculated using a theoretical model by Gouy-Chapman ${ }^{12}$ at $25^{\circ} \mathrm{C}$ as follows:

$$
k=2.32 \times 10^{9}\left(\Sigma c_{i} z_{i}^{2}\right)^{1 / 2},
$$

where $1 / k$ is the double-layer thickness, $c_{i}$ is the ion concentration, and $z_{i}$ determines the extent of counterion charge in the solution. The final particle sizes are calculated to be 22 and $27 \mathrm{~nm}$, for 10 and $20 \mathrm{~nm}$ respectively, which agrees with experimental results.

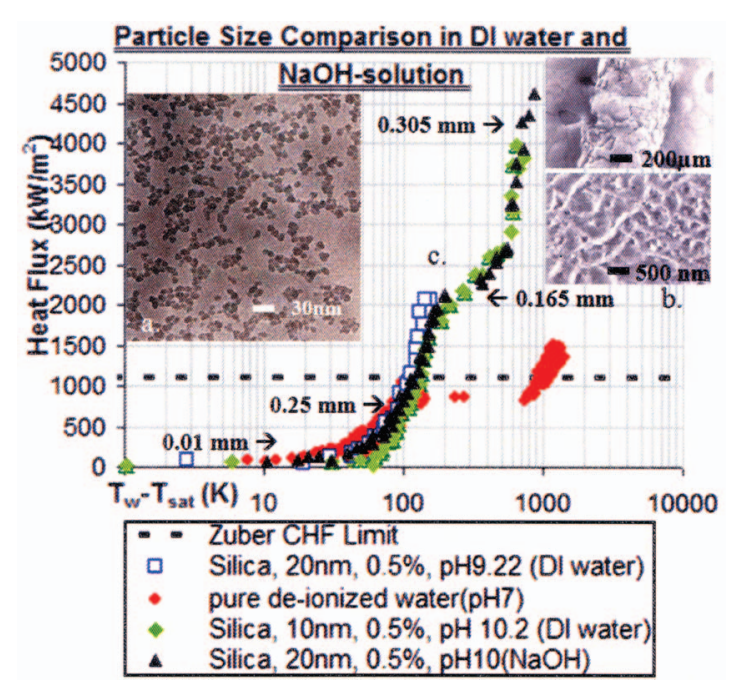

FIG. 4. (Color) (a) Colloidal behavior of silica in deionized water as seen in TEM; (b) TEM and SEM pictures of the Nichrome wire after deposition of silica; (c) Pool boiling CHF for 10 and $20 \mathrm{~nm}$ particles in deionized water with no additives and $\mathrm{NaOH}$. Different levels of deposition on the wire are shown in $\mathrm{mm}$ at different stages of boiling.
Two characteristics of silica nanoparticles are explored: one is the role of the salts and the other is the effect of particle size on critical heat flux. In the presence of salts (such as in the buffer solution), due to van der Waals attractive forces, silica tends to form polymers, i.e., a chain of silicate layers, representing clusters and films. Since buffer solutions are weak electrolytes, they do dissociate at a very low rate when current is passed. Even so, they can release some sodium and potassium ions which could bond to the silica clusters and act as ion conductors. Thus, they form a solid hybrid polymer, with $\mathrm{Na}+$ ion being more probable to bond because of its smaller size. Both 10 and $20 \mathrm{~nm}$ particles become neutrally charged. Although $\mathrm{CHF}$ is still significantly increased compared to pure fluids, the clustered particles result in a detrimental performance compared to silica suspended in deionized water, as noted in Fig. 3.

In Fig. 4, with no salts present (i.e., $\mathrm{SiO}_{2}+$ deionized water and $\mathrm{NaOH}$ solution), there are no significant agglomerations. The electrolytes present in the solution are responsible for carrying part of the applied current. The charged silica particle in suspension experiences "hydration" of the surface of the particles, i.e., a layer of water molecules are hydrogen bonded to $\mathrm{SiOH}$ groups. The corresponding zeta potential values for the two particle sizes are in the neighborhood of $-20 \mathrm{mV}$, as shown in another study, hence negative charge barriers exist. The 10- and 20-nm particles grow to 19 and $22 \mathrm{~nm}$, respectively, in DI water.

The CHF for $20 \mathrm{~nm}$ in a strong electrolyte solution $(\mathrm{NaOH})$ goes up to $4600 \mathrm{~kW} / \mathrm{m}^{2}$ (Fig. 4) with an increase in the size of the particle which is negatively charged. Having an overall negative charge and a thin double layer, particles bond to a limited amount of free ions, leaving the majority in the solution. Therefore, it is reasonable to expect high potential transport through the liquid and diversion of a significant amount of current from the $\mathrm{NiCr}$ wire, giving it extended life. The double diffuse layer on the particle gets distorted and attracted to the wire, resulting in significant deposition. This surface porosity is also responsible for increasing the heat transfer. Heat transfer results were obtained for $0.2 \%$ with no additives. An increase of $10-15 \%$ in CHF was observed for $0.5 \%$ solution compared to $0.2 \%$.

The authors acknowledge financial support from the National Science Foundation Grant No. NER 0404174.

${ }^{1}$ J. A. Eastman, S. U. S. Choi, S. Li, W. Yu, and L. J. Thompson, Appl. Phys. Lett. 78, 718 (2001).

${ }^{2}$ S. P. Jang and S. U. S. Choi, Appl. Phys. Lett. 84, 4316 (2004).

${ }^{3}$ P. Vassallo, R. Kumar, and S W. D'Amico, Int. J. Heat Mass Transfer 47, 407 (2004)

${ }^{4}$ P. Bhattacharya, S. K. Saha, A. Yadav, P. E. Phelan, and R. S. Prasher, J. Appl. Phys. 95, 6492 (2004).

${ }^{5}$ P. Keblinski, S. R. Phillpot, S. U. S. Choi, and J. A. Eastman, Int. J. Heat Mass Transfer 45, 855 (2002).

${ }^{6}$ N. R. Jana, Y. Chen, and X. Peng, Chem. Mater. 16, 3931 (2004).

${ }^{7}$ www.resistancewire.com.

${ }^{8}$ N. Zuber, Int. J. Heat Mass Transfer 6, 53 (1963).

${ }^{9}$ W. M. Rohsenow, Trans. ASME, Ser. C: J. Heat Transfer 84, 969 (1962).

${ }^{10} \mathrm{http} / / / \mathrm{www} . r \mathrm{rc}$. state.tx.us/divisions/afred/education/lessonpdf/ 3_3electrolysis.pdf.

${ }^{11}$ R. K. Iler, Surface and Colloid Science, edited by E. Matijevic (Wiley, New York, 1973), Vol. 6.

${ }^{12}$ M. Elimelech, J. Gregory, X. Jia, and R. A. Williams, Particle Deposition and Aggregation (Butterworth and Heinemann, Wooburn, MA, 1995). 